\title{
Eigenschaftsanalyse zur Entwicklung additiv gefertigter Prototypen - Das House of Properties
}

\author{
Stefan Schork ${ }^{1} \cdot$ Kai Güttinger ${ }^{1}$ Eckhard Kirchner ${ }^{1}$ (D) \\ Eingegangen: 27. Februar 2020 / Angenommen: 18. August 2020 / Online publiziert: 16. September 2020 \\ (c) Der/die Autor(en) 2020
}

\section{Zusammenfassung}

In Zeiten dynamischer Märkte und internationalen Wettbewerbs erlangt die Verkürzung von Produktentwicklungszyklen immer größere Bedeutung. Gleichzeitig werden auch die Möglichkeiten zur Herstellung von Prototypen zur Unterstützung der Produktentwicklung immer zahlreicher und diverser. Dieser Artikel beschäftigt sich mit der Ermittlung von Anforderungen an funktionelle Prototypen. Mithilfe dieser Prototypen sollen verlässliche Verifikationen der Einhaltung von Anforderungen an verschiedene Produktfunktionen ermöglicht werden. Als Beispiel werden dazu Produkte herangezogen, die im Spritzgussverfahren aus verschiedenen Kunststoffen hergestellt werden. Die konventionelle Verifikation dieser Produkte ist durch die Fertigung und den Einsatz von Prototypenwerkzeugen sehr zeitaufwändig und kostenintensiv. Der Einsatz additiver Fertigungsverfahren bietet die Möglichkeit, diese Faktoren zu senken. Hierbei muss jedoch sichergestellt sein, dass die Ergebnisse der Tests mit additiv gefertigten Prototypen auch auf das spritzgegossene Produkt übertragbar sind. Durch die Änderung des Fertigungsverfahrens und des verwendeten Materials treten Änderungen gewisser Eigenschaften des Prototyps im Vergleich zum finalen Produkt auf. Diese Abweichungen können sich wiederum negativ auf die Aussagefähigkeit der Ergebnisse der Verifikation mittels Prototypen auswirken, wenn die Eigenschaften essentiellen Einfluss auf die zu verifizierenden Funktionen haben. Daher wurde eine Methode - das House of Properties - entwickelt, mit der zum einen der Bedarf an Prototypen ermittelt werden kann und zum anderen die Eigenschaften des in der Entwicklung befindlichen Bauteils dahingehend untersucht werden können, welche Eigenschaften besonders kritisch auf bestimmte Funktionen wirken. Durch diese Analyse können Anforderungen an additiv gefertigte Prototypen identifiziert werden, die als Basis zur Auswahl des Fertigungsverfahrens und des Materials dienen. Die entwickelte Methode wird im Rahmen dieses Artikels anhand eines Industriebeispiels evaluiert und weiter erläutert.

Stefan Schork

office@pmd.tu-darmstadt.de

1 Fachgebiet für Produktentwicklung und Maschinenelemente,

Technische Universität Darmstadt, 64287 Darmstadt,

Deutschland 


\title{
Analysis of properties for the development of additively manufactured prototypes-the House of Properties
}

\begin{abstract}
In times of dynamic markets and international competition, the shortening of product development cycles is becoming increasingly important. At the same time, the possibilities of producing prototypes to support the product development are becoming more numerous and diverse. This article deals with the determination of requirements for functional prototypes. The aim of these prototypes is to facilitate the reliable verification of meeting the requirements of different product functions. As an example, products that are planned to be manufactured using plastic injection moulding are used. The conventional verification of these products is very time-consuming and cost-intensive due to the production and use of prototype tools. The use of additive manufacturing offers the possibility to reduce these downsides. However, it must be ensured that the results of tests with additively manufactured prototypes are transferable to the injection-moulded product. By changing the manufacturing process and the material used, changes in certain properties of the prototype compared to the final product occur. These deviations can in turn have a negative effect on the meaningfulness of the results of the verification using prototypes if the deviating properties have an essential influence on the functions to be verified. For this reason, a method - the House of Properties-is introduced in this work, with which, the need for prototypes can be determined on the one hand and, on the other hand, the properties of the product under development can be examined to determine which properties have a particularly critical effect on certain functions. This analysis can be used to identify requirements for additively manufactured prototypes, which serve as a basis for selecting the manufacturing process and material. The developed method is evaluated and further explained in this article using an industrial example.
\end{abstract}

\section{Motivation}

Immer kürzere Produktlebenszyklen stellen besondere Herausforderungen in der Produktentwicklung dar. Diese vermehrt festzustellende Zyklusverkürzung hat eine immer kürzere Zeitspanne zwischen der Entwicklung eines Produkts und des Anlaufens der Serienfertigung zur Folge. Daraus ergeben sich auch hinsichtlich der Entwicklungsprozesse und des Risikomanagements verschärfte Anforderungen.

Insbesondere bei Produkten, deren Komponenten und Bauteile komplett oder teilweise mittels Kunststoffspritzgussverfahren hergestellt werden, stellt die Beschaffung beziehungsweise die Herstellung der Werkzeuge einen kritischen Aspekt im Zeitplan dar. Je nach Komplexität des Bauteils werden dafür üblicherweise zwischen acht und 20 Wochen benötigt [1]. Neben dem zeitlichen Rahmen ist auch der finanzielle Aufwand hoch. Entsprechend sind Fehler und Verzögerungen, beispielsweise durch Konstruktionsmängel des Bauteils, besonders kritisch.

Aus diesen Gründen wird versucht, die Verifikation der Bauteile hinsichtlich der gestellten Anforderungen mithilfe von Prototypen so weit wie möglich vor den Bau der Serienwerkzeuge zu verschieben. Dadurch kann das terminliche und finanzielle Risiko bereits in der Entwicklung erheblich minimiert werden. Die Folgekosten solcher Fehler werden mit der sogenannten „Rule of Ten“ beschrieben. Sie besagt, dass jede Phase im Produktlebenszyklus, in welcher der Fehler nicht erkannt und behoben wird, eine Verzehnfachung der Kosten zur Korrektur zur Folge hat [2].
Die Verwendung von funktionellen Prototypen ermöglicht eine solche zeitliche Verlagerung der Bauteilverifikation vor die Herstellung der Spritzgusswerkzeuge, stellt allerdings an anderer Stelle eine besondere Herausforderung dar. Zur Herstellung physischer Prototypen im Spritzgussverfahren, werden Prototypenwerkzeuge benötigt, die zwar durch die geringeren Stückzahlen und damit geringere geforderte Lebensdauer mit geringerem Aufwand im Vergleich zum Serienwerkzeug gefertigt werden können, jedoch weiterhin mehrere Wochen zur Fertigung benötigen. Daher bietet sich die Prototypenfertigung mittels additiver Verfahren an, da mit ihnen auch komplexe Bauteile in kurzer Zeit und mit niedrigen Stückkosten hergestellt werden können. Im Vergleich dazu liegen die Kosten pro Bauteil spritzgegossener Bauteile bei niedrigen Stückzahlen auf einem hohen Niveau, wie Abb. 1 schematisch verdeutlicht [2].

Da die Eigenschaften eines spritzgegossenen Kunststoffbauteils allerdings stark von den Verfahrensbedingungen, der exakten Werkstoffspezifikation und anderen Faktoren abhängig sind, besteht die Gefahr, dass sich die Eigenschaften des finalen Bauteils und dessen Prototypenunterscheiden. Ist dies der Fall, ist ein direkter Übertrag der mit Prototypen durchgeführten Untersuchungen auf das finale Bauteil nicht möglich. Die vollständige Verifikation der Entwicklung ist ebenfalls nur durch die Verwendung von zumindest seriennahen Bauteilen gewährleistet [3].

Da additive Fertigungsverfahren großes Potenzial für den aufgezeigten Anwendungsfall bieten [3], soll deren Anwendbarkeit durch die entwickelte Methode vereinfacht werden. Mithilfe der Methode lassen sich die Bedar- 


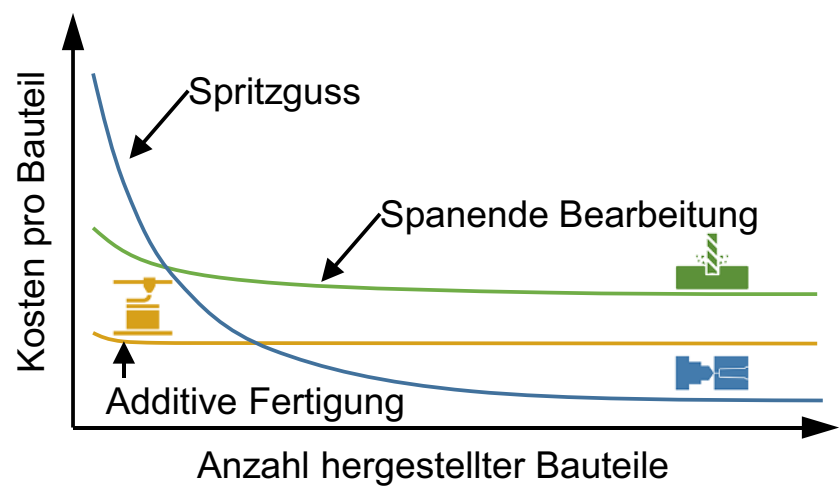

Abb. 1 Schematische Betrachtung der Kosten pro Bauteil in Abhängigkeit zur Anzahl der hergestellten Bauteile in Anlehnung an [2]

fe für Prototypen ermitteln und Handlungsempfehlungen hinsichtlich Auswahl geeigneter Fertigungsverfahren und möglichen Vereinfachungen des Prototyps abhängig vom spezifischen Anwendungsfall geben. Im Rahmen dieses Artikels wird die Methode anhand von Kunststoffspritzgussbauteilen erläutert, da in diesem Bereich der größte positive Effekt durch die Verkürzung der Vorlaufzeit zwischen Entwicklung und Einsatz von Prototypen besteht. Die Methode eignet sich allerdings auch für weitere Anwendungsfälle.

Beim Einsatz von Methoden in der Entwicklung existieren mehrere Hürden [4]. Daher basiert die Methode auf einer intuitiven und offenen Form, um eine gute Anwendbarkeit sicherstellen zu können. Um beispielsweise die Auswirkungen fehlenden Methodenwissens gering zu halten, orientiert sich die Methode an etablierten Methoden wie dem Quality Function Deployment (QFD), das zur Steigerung der Produktqualität und Abdeckung der Kundenwünsche eingesetzt wird oder der Failure Mode and Effects Analysis (FMEA), die zur Analyse von Produkten hinsichtlich möglicher auftretender Fehler und deren Vermeidung angewandt wird.

Die durchgeführten Bauteilanalysen erfordern einen technischen Hintergrund und eine gewisse Erfahrung bei der Entwicklung von Produkten sowie die Kenntnis der Prozesse beim Kunststoffspritzguss. Die verschiedenen Entscheidungsgrundlagen sollen zur weiteren Reduktion des Arbeitsaufwandsmittels der Methode ohne Mehraufwand dokumentiert und so auf Dauer verfügbar gemacht werden [4].

\section{Funktionsanalyse}

Basierend auf einer geklärten Aufgabenstellung werden im Rahmen der Produktentwicklung unterschiedliche Lösungskonzepte und Entwürfe erarbeitet [5]. Diese werden im Anschluss hinsichtlich ihrer Eignung und der Erfüllung der
Anforderungen untersucht. Dabei ist es unerheblich, in welchem Detaillierungsgrad sich die Entwürfe befinden. Je früher festgestellt werden kann, dass der Entwurf gewisse Anforderungen nicht erreicht, desto geringer sind zeitliche und finanzielle Aufwände zur Behebung dieses Zustands.

Zur Identifikation von Funktionen und Wechselwirkungen, die eine kritische Auswirkung auf die Funktionsfähigkeit des Entwurfs haben, wird zunächst eine Funktionsanalyse durchgeführt. Die Analyse der Produktfunktionen ist notwendig, da ein Prototyp ein Abbild des entwickelten Entwurfs darstellt, dessen Anforderungen sich von den Anforderungen an das Produkt in Entwicklung unterscheiden. Entsprechend werden die Produktfunktionen im Prototyp abgebildet.

Die Funktionsanalyse stellt somit die Grundlage für die spätere Bauteilanalyse und den Übertrag auf den Prototyp dar. Die Funktionsanalyse soll zum einen das Verständnis für das Produkt verbessern und zum anderen eine Bewertung der Kritikalität der Bauteilfunktionen ermöglichen. Diese Kritikalitätsbewertung soll dabei in erster Linie den Verifikationsbedarf klären und aufzeigen, welche Anforderungen an Funktionen mithilfe von Prototypen in einem frühen Entwicklungsstadium verifiziert werden sollen.

Wie bereits beschrieben, leiten sich sowohl die Gesamtfunktion als auch die Teilfunktionen aus dem jeweiligen Entwurf für ein Produkt ab. Jede Teilfunktion eines Produkts hat Einfluss auf die Gesamtfunktion. Die Einflussstärke jeder Teilfunktion hängt allerdings stark vom betrachteten Anwendungsfall beziehungsweise vom Verwendungszweck des ab. Somit muss ein Prototyp in der Regel nur einen Teil der Funktionen abbilden, während weniger relevante Funktionen gegebenenfalls vernachlässigt oder zumindest vereinfacht dargestellt werden können.

Besonders die Einhaltung der Anforderungen an kritische Funktionen sollte zur Minimierung der Entwicklungsrisiken früh verifiziert werden [6]. Um diese Kritikalität der Funktionen bewerten zu können, werden diese hinsichtlich ihrer Neuheit, ihrer technischen Schwierigkeit und ihrer Bedeutung für das Endprodukt bewertet, in Anlehnung an [7]. Dadurch spannt sich der in Abb. 2 dargestellte Raum auf. Diese Gliederung macht eine objektive Analyse der Kritikalität möglich und dient als Grundlage für die späteren Ausarbeitungen.

\subsection{Neuheit}

Da Prototypen insbesondere dann einen großen Wert für die Produktentwicklung haben, wenn neue oder unbekannte Funktionen integriert werden sollen, wird zunächst die Neuheit bewertet. Eine niedrige Bewertung (1) beschreibt eine gut bekannte Funktion, mit der bereits Erfahrungen in einem zumindest sehr ähnlichen Anwendungsfall gemacht wurden. Eine hohe Bewertung (3) wird hingegen dann ver- 


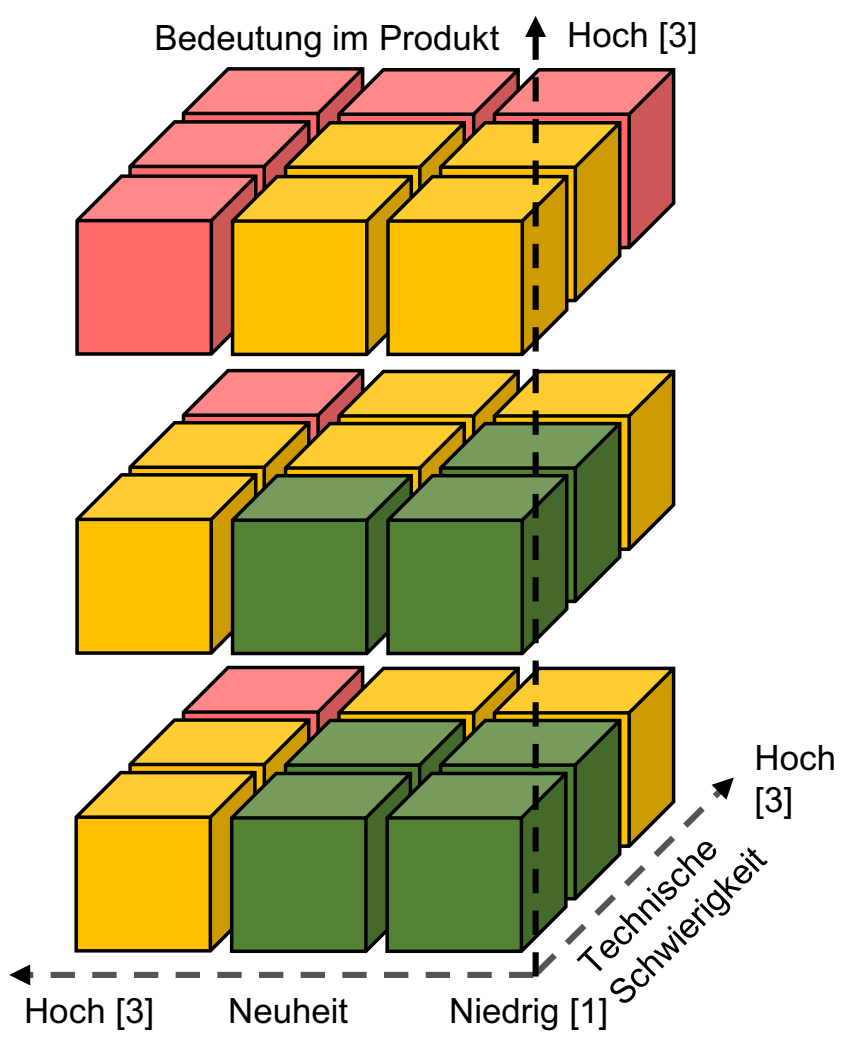

\section{Unkritisch Sehr Kritisch}

Abb. 2 Bewertung der Kritikalität verschiedener Funktionen in Anlehnung an [7]

geben, wenn die Funktion alleine oder im Zusammenspiel mit weiteren Funktionen weitgehend unbekannt ist. Somit kann auf keine weitreichende Erfahrung auch bezüglich der Wechselwirkungen zurückgegriffen werden.

\subsection{Technische Schwierigkeit}

Die Feststellung der technischen Schwierigkeit ermöglicht die gezielte Betrachtung von anspruchsvollen Funktionen und Wechselwirkungen. Hierzu wird, ähnlich wie im House of Quality, bewertet, wie hoch der erwartete Aufwand ist, um diese Funktion im Produkt abzubilden. Dabei entspricht eine hohe Bewertung (3) einer hohen technischen Schwierigkeit und somit weitreichenden Auswirkungen auf das Produkt, zum Beispiel, wenn komplexe Geometrien mit engen Toleranzen hergestellt werden müssen. Eine niedrige Bewertung (1) wird dann vergeben, wenn die Funktionen mit geringem Aufwand im Produkt abgebildet werden können und die Wechselwirkungen klar ersichtlich sind.

\subsection{Auswirkungen im Endprodukt}

Zur Ermittlung der Kritikalität einer Funktion sollten auch die Auswirkungen bei teilweiser oder vollständiger Nichterfüllung mit einbezogen werden. Die Bedeutungshöhe einer solchen Fehlfunktion kann beispielsweise durch eine FMEA ermittelt werden. Ähnlich wie bei der Neuheit und der technischen Schwierigkeit entspricht eine hohe Wertung (3) einer starken Auswirkung im Produkt, während eine niedrige Bewertung (1) bei sehr geringen negativen Auswirkungen vorzusehen ist.

\subsection{Bewertung der Kritikalität einer Funktion}

Die Kritikalität einer Funktion kann nun anhand der drei Kategorien bewertet werden. Eine niedrige Kritikalität (grün) einer Funktion liegt dann vor, wenn maximal zwei der Einzelwertungen eine mittlere Bedeutung haben. Die Funktion hat eine mittlere Kritikalität (gelb), wenn maximal eine der Einzelwertungen eine hohe Bedeutung hat. Sobald zwei hohe Einzelwertungen vorliegen, kann von einer hohen Kritikalität (rot) gesprochen werden. Entsprechend sollte die Einhaltung der Anforderungen an diese Funktion in einem frühen Stadium verifiziert werden.

\section{Bauteilanalyse}

Nach der Identifikation kritischer Funktionen steht der Nachweis der Funktionsfähigkeit des Entwurfs im nächsten Schritt im Vordergrund. Dazu werden in der Regel unterschiedliche Prototypen eingesetzt. Der Übertrag der Konstruktion auf funktionsfähige Prototypen stellt durch die zahlreichen Besonderheiten von Kunststoffspritzgussbauteilen und die beim Kunststoffspritzgießen induzierten Eigenschaften eine besondere Herausforderung dar. Entsprechend wird zunächst eine zielgerichtete Analyse der Eigenschaften der spritzgegossenen Bauteile des Produkts erfolgen, die im nächsten Schritt eine Abbildung im Prototyp ermöglichen. Bauteile beschreiben dabei Elemente, die nicht ohne Verlust der grundlegenden Eigenschaften weiter zerlegt werden können [8].

Zur Gliederung der Eigenschaften des Bauteils sieht die VDI 2223 eine Einteilung in Gestalt- und Werkstoffeigenschaften vor [9]. Hiernach wird die Gestalt beispielsweise durch die Form, Abmessungen, Anordnung und Anzahl der Gestaltelemente beschrieben. Jedes Element ist also auf unterschiedliche Gestalteigenschaften reduzierbar. Des Weiteren wird die Beschaffenheit der Werkstoffeigenschaften beschrieben. Diese sind laut VDI 2223 unter anderem die Werkstoffe an sich, jedoch (falls vorhanden) auch die Betriebsstoffe der Gestaltelemente [9, 10]. 
Im Rahmen dieser Ausarbeitung wird die Unterteilung dahingehend erweitert, dass die Gestalteigenschaften wiederum in die beiden Punkte Geometrische Eigenschaften und Oberflächeneigenschaften unterteilt werden. Die in der VDI 2223 beschriebenen Werkstoffeigenschaften werden auch hier übernommen. Die Eigenschaften, die der zu entwickelnde Prototyp abbilden muss, ergeben sich dabei aus den Bauteilentwürfen.

\subsection{Werkstoffeigenschaften}

Die Werkstoffeigenschaften beschreiben neben den mechanischen Eigenschaften des Werkstoffs auch weitere Spezifikationen wie die Brennbarkeit, die Medienbeständigkeit, die Permeabilität gegenüber verschiedenen Medien, das Verhalten bei unterschiedlichen Einsatztemperaturen oder die Optik. Im Gegensatz zur Definition der VDI 2223 werden in dieser Ausarbeitung die Eigenschaften der Werkstoffoberfläche nicht direkt zu den Werkstoffeigenschaften gezählt, sondern separat beschrieben[9]. Dies wird damit begründet, dass Oberflächen im Sinne von Wirkflächen eine zentrale Rolle einnehmen.

\subsection{Oberflächeneigenschaften}

Die Eigenschaften der Bauteiloberfläche sind dahingehend von besonderer Bedeutung, dass die Oberflächen in direktem Wirkkontakt entweder mit anderen Bauteilen beziehungsweise Formelementen oder mit der Umgebung sind. Die Umgebungsbedingungen wirken über die Oberfläche auf das Bauteil ein und können dadurch die Bauteileigenschaften beeinflussen oder verändern. Neben physischen Eigenschaften wie der Rauheit oder der Härte spielen mithin auch die Wechselwirkungen mit einem umströmenden Medium eine Rolle. Darüber hinaus legt die Oberflächenbeschaffenheit in besonderem Maße fest, welche Auswirkungen ein Wirkkontakt zwischen mehreren Formelementen auf diese hat. Beispielsweise seien hier das Gleitverhalten oder der Oberflächenverschleiß bei Reibkontakt genannt. Zur Identifikation der Wirkkontakte biete sich unter anderem das Contact and Channel Model an [11].

\subsection{Geometrische Eigenschaften}

Die geometrischen Eigenschaften eines Bauteils beschreiben zum einen seine äußere Geometrie, zum anderen seine Toleranzgrenzen bei der Fertigung. Funktionskritische Maße sind in der Konstruktion der Bauteile immer mit Toleranzangaben zu versehen [12]. Entsprechend müssen auch die Prototypen diese Toleranzgrenzen einhalten, um die vorgesehenen Funktionen möglichst abbildtreu ausführen zu können. Außerdem kann hierdurch bereits im Vorfeld zur Fertigung die korrekte Lage und Angabe der Toleranzen durch Prototypen überprüft werden. Dabei ist zu beachten, dass bei Herstellung der Bauteile im Spritzgussverfahren mit Verzug und Schwindung beim Abkühlen der Bauteile gerechnet werden muss. Die Korrekte Einstellung der Verfahrensparameter kann an dieser Stelle jedoch nicht durch einen additiv gefertigten Prototyp untersucht werden. Überprüft wird daher lediglich, ob das festgelegte Toleranzfeld zur Erfüllung der Funktion führt.

\subsection{Umgebungsbedingungen}

Bauteile sind, unabhängig von Herstellverfahren, Geometrie und Werkstoff, unterschiedlichen Umgebungseinflüssen ausgesetzt. Diese wirken sich wiederum positiv oder negativ auf die Eigenschaften der Bauteile aus. Die Auswirkungen der Umgebungsbedingungen können allerdings oftmals nicht ohne großen Aufwand qualitativ ermittelt werden, weshalb sie nicht gesondert berücksichtigt werden. Vielmehr können Wechselwirkungen mit der Umwelt nur mit den realen Parametern am Bauteil abgebildet werden. Aus diesem Grund sollen die Umgebungsbedingungen und deren Auswirkungen auf das Bauteil nicht näher betrachtet werden.

\section{Eigenschaftsanalyse im House of Properties}

Im Folgenden wird das House of Properties (HoP) eingeführt. Damit kann unter anderem die vorangegangene Funktionsanalyse mit der eigentlichen Bauteilanalyse kombiniert werden. Ziel der Eigenschaftsanalyse ist es, herauszufinden, welche Eigenschaften einen kritischen Einfluss auf die Funktion des Bauteils haben. Kritische Eigenschaften sollten in einem funktionellen Prototyp nur geringfügig abweichen, während auf die exakte Abbildung unkritischer Eigenschaften gegebenenfalls verzichtet werden kann.

Angelehnt an das House of Quality, welches in erster Linie dazu eingesetzt wird, die Anforderungen und Erwartungen des Kunden in Beziehung zu den Entwurfsmerkmalen zu setzen, um Entwicklungsschwerpunkte und technische Anforderungen zu identifizieren, ist das House of Properties wie in Abb. 3 zu sehen aufgebaut.

Neben den eigentlichen Bauteilfunktionen und -konfigurationen $^{1}$ (a.) werden zunächst wichtige Qualitätsanforderungen und Rahmenbedingungen (b.) in das House of Properties eingetragen. $\mathrm{Zu}$ den Bauteilfunktionen wird nun die jeweilige Kritikalität dieser Funktion mittels des in Abschn. 2 erläuterten Vorgehens ermittelt und einge-

\footnotetext{
${ }^{1}$ Eine Konfiguration kann eine Funktion detaillierter beschreiben. Sie stellt entweder eine Unterfunktion oder eine Anforderung an die Funktion dar [13].
} 


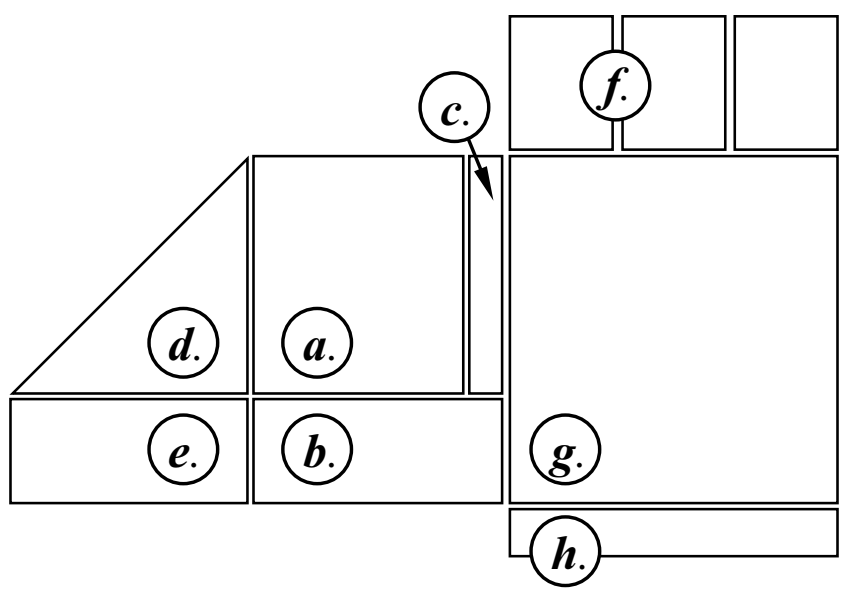

Abb. 3 Schematische Darstellung des House of Properties (Erläuterungen im Text)

tragen (c.). Anschließend werden die Wechselwirkungen der Bauteilfunktionen untereinander hinsichtlich ihrer Einflussstärke bewertet (d.). Dabei ist darauf zu achten, dass sowohl negative als auch positive Beeinflussungen beziehungsweise Wechselwirkungen berücksichtigt werden müssen. Die Wechselwirkungen zwischen den Funktionen und den Rahmenbedingungen werden auf gleiche Weise in das entsprechende Feld eingetragen (e.). Dies ermöglicht im Weiteren die Identifikation von wichtigen Wechselwirkungen im Bauteil. Somit kann vermieden werden, dass Funktionen isoliert untersucht werden, die besonders durch das Zusammenspiel mit anderen Funktionen kritische Auswirkungen auf das Produkt haben.

Zentrales Element des House of Properties ist die Analyse der Einflussstärke der Bauteile und Funktionselemente auf die verschiedenen Funktionen. Dazu werden zunächst die Bauteile beziehungsweise Funktionselemente eines Produkts oder eines funktionell abtrennbaren Teilsystems dieses Produkts im oberen Bereich des House of Properties vermerkt.

Die Bauteile werden dabei nicht im Ganzen hinsichtlich verschiedener Auswirkungen auf die Bauteilfunktionen untersucht, sondern in den beschriebenen Kategorien Werkstoffeigenschaften, Oberflächeneigenschaften und Geometrische Eigenschaften (f.). Diese drei Kategorien sind jeweils unter den Bauteilbezeichnungen abgebildet. Dabei soll lediglich eine intuitive Bewertung der Einflussstärken erfolgen, da die absoluten Eigenschaften des finalen Produkts in einem frühen Entwicklungsstadium kaum bis gar nicht beurteilbar sind. Entsprechend soll die Einflussstärke zunächst nur abgeschätzt werden.

Die Analyse der Bauteileigenschaften bezieht sich dabei weniger auf die tatsächlichen Eigenschaften des Bauteils als auf deren Einflussstärke auf verschiedene Funktionen. Dies ermöglicht die Betrachtung der Anforderungen an einen Prototyp hinsichtlich seines spezifischen Einsatzzwecks. In der Regel ist es nicht notwendig alle Eigenschaften des Bauteils in einem Prototyp mit gleicher Exaktheit abzubilden. Vielmehr ist die Bedeutung sowohl auf Bauteilebene als auch auf Eigenschaftsebene stark vom Einsatzzweck abhängig. Um Prototypen mit geringstmöglichem Aufwand mit einer möglichst präzisen Nachbildung der Eigenschaften herstellen zu können, muss entsprechend analysiert werden, welchen Einfluss diese auf bestimmte Funktionen haben. Dabei sind besonders die durch das Spritzgussverfahren induzierten Eigenschaften, beispielsweise die Anisotropie faserverstärkter Kunststoffe durch Ausrichtung der Fasern während des Spritzgießens zu beachten.

Die eigentliche Bewertung wird in einer Matrix ( $g$.), ähnlich dem House of Quality mit einer progressiven Bewertungsskala, durchgeführt, um die Unterschiede besser hervorzuheben [14]. Diese Skala umfasst die Wertungen kein Einfluss (0), geringer Einfluss (1), deutlicher Einfluss (2) und starker Einfluss (4). Die progressive Skala wird in dieser Abstufung gewählt, um den Fokus hin zu starken Einflüssen zu lenken, ohne dabei einzelne Einflüsse zu deutlich zu repräsentieren, was bei einer Verwendung der aus dem House of Quality bekannten Abstufung (0-1-3-9) zu befürchten ist. Die eigentliche Bewertung beruht dabei hauptsächlich auf Erfahrungswerten desjenigen, der sie durchführt. Ist es dabei nicht möglich, den Einfluss abzuschätzen, sollte von einem deutlichen oder starken Einfluss ausgegangen werden. Generell sollte der Einfluss eher überschätzt als unterschätzt werden. So kann das Risiko des Nichtentdeckens von Fehlern reduziert werden.

Als generelle Aussage über die Bedeutung und Einflussstärke einer Eigenschaft wird die $\mathrm{HoP}$-Zahl gebildet $(h$.). Je höher sie ist, desto einflussreicher ist die entsprechende Eigenschaft im spezifischen Anwendungsfall. Die $\mathrm{HoP}$-Zahl und somit die Aussage, welche Eigenschaften für einen bestimmten Prototyp welche Einflussstärke haben, ist immer davon abhängig, welche Funktionen in ihn integriert werden sollen. Entsprechend muss eine Funktionsauswahl durchgeführt werden, für die im House of Properties die individuelle Bewertung der jeweiligen Eigenschaftsbedeutung erfolgt. Eigenschaften, welche mit einer hohen $\mathrm{HoP}$ Zahl belegt wurden, sollten sowohl in einem physischen als auch in einem virtuellen Prototyp möglichst realitätsnah abgebildet werden. Eine niedrige $\mathrm{HoP}$-Zahl lässt hingegen darauf schließen, dass die spezifischen Eigenschaften durch weniger genaue Abbildverfahren dargestellt werden können ohne das Verifikationsergebnis zu beeinträchtigen.

\section{Beispiel}

Im Folgenden soll die Erstellung des House of Properties anhand eines Beispiels näher erläutert werden. Dabei wer- 


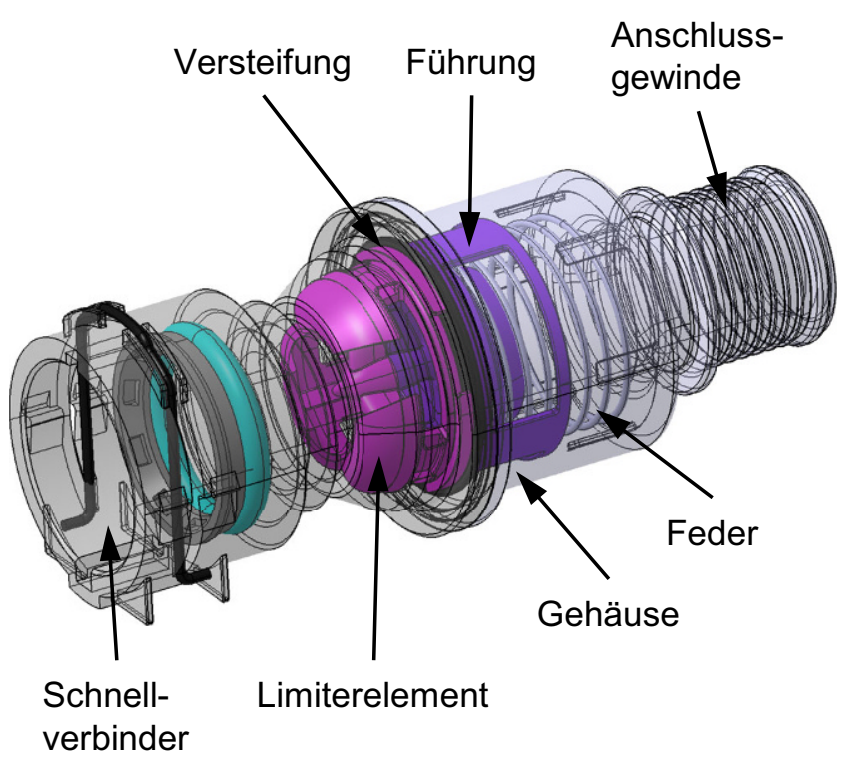

Abb. 4 CAD-Modell des Ventils zur Reduktion des Volumenstroms

den aus Gründen der Übersichtlichkeit nicht alle Ergebnisse dargestellt.

\subsection{Entwurf}

In Kooperation mit Illinois Tool Works (ITW) wurde ein passives Ventil entwickelt, das beispielsweise im Kühlwasserkreislauf eines Fahrzeugs eingebaut werden kann. Das Ventil stellt dahingehend eine Neuheit dar, dass es bei einem hohen Volumenstrom schließt und somit einen erhöhten Druckverlust generiert, was wiederum dazu führt, dass der Volumenstrom sinkt. Diese Funktion ermöglicht eine effizientere Auslastung der Wasserpumpe und somit eine geringere Energieaufnahme, besonders bei hohen Motordrehzahlen. Der Entwurf ist dabei wie in Abb. 4 dargestellt aufgebaut. Die Funktion des volumenstromlimitierenden Elements (Limiterelement) ist in Abb. 5 verdeutlicht.

\subsection{Funktionsanalyse}

Der Entwurf weist unter anderem die in Abb. 6 dargestellten Funktionen und Konfigurationen auf. Über die jeweilige Bewertung von Neuheit, technischer Schwierigkeit und Auswirkung im Produkt kann die entsprechende Kritikalität ermittelt werden. Diese ist für einige der Funktionen besonders hoch. Auch verschiedene Randbedingungen und deren Wechselwirkungen mit den Funktionen sind abgebildet.

\subsection{House of Properties}

Die Ergebnisse der Funktionsanalyse sind auf der linken Seite im House of Properties in Abb. 6 (oben) abgebildet. Die Gliederung des Produkts in Bauteile beziehungsweise Funktionselemente kann aus der vorangegangenen Konzeptentwicklung übernommen werden.

Die eigentliche Bewertung der Einflussstärke der Bauteileigenschaften auf die Funktionen wird in der rechtsstehenden Matrix durchgeführt. Basierend auf dem aktuellen Entwicklungsstand und dem entsprechenden Verständnis für das Produkt wird hier die jeweilige Einflussstärke einer Eigenschaft auf Funktionen untersucht. Die Schlüsse, welche daraus gezogen werden können, werden im Folgenden diskutiert.
Abb. 5 Darstellung der Funktionsweise des Limiterelements im Inneren des Ventils. a Offen b Geschlossen
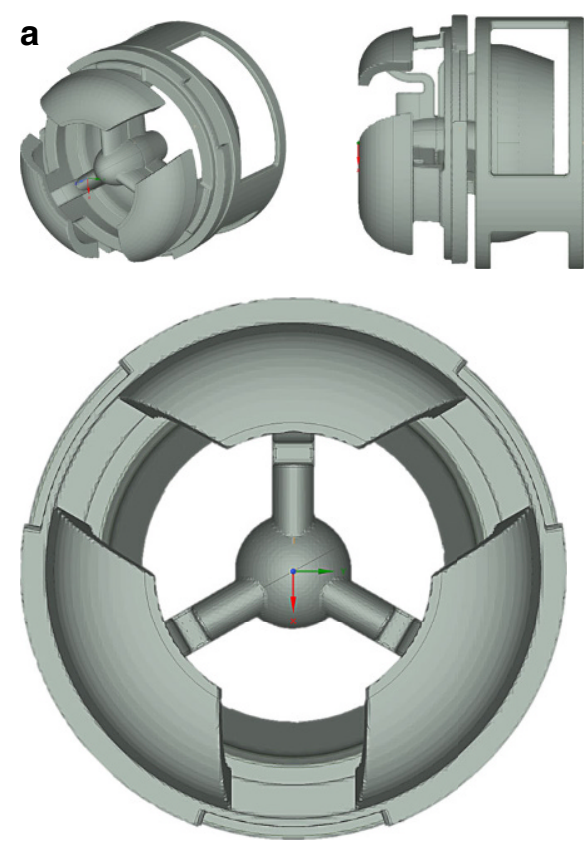
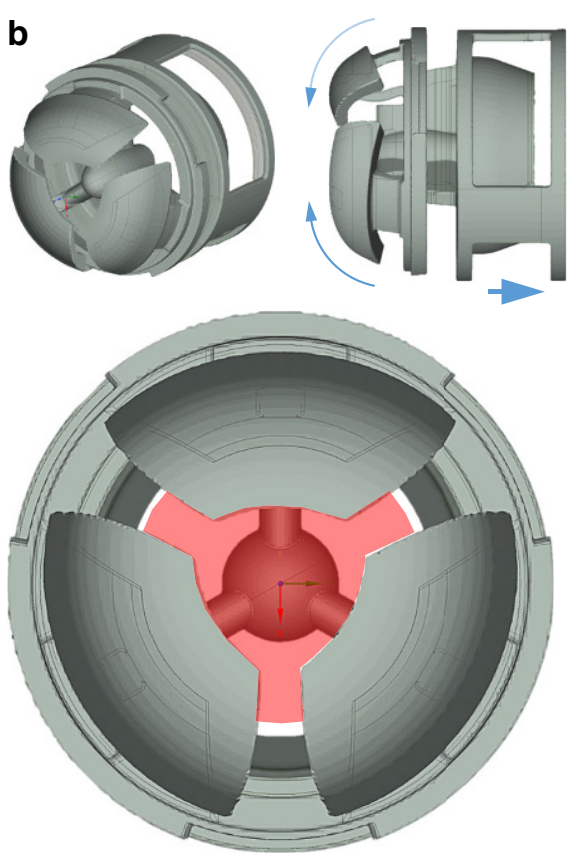
Abb. 6 Bewertung der Kritikalität der verschiedenen Funktionen im House of Properties (oben, linke Seite des House of Properties) und Bewertung des Einflusses verschiedener Eigenschaften auf die Funktionen des Produkts (unten, rechte Seite des House of Properties), die verschiedenen Bereiche sind gemäß Abb. 3 gekennzeichnet

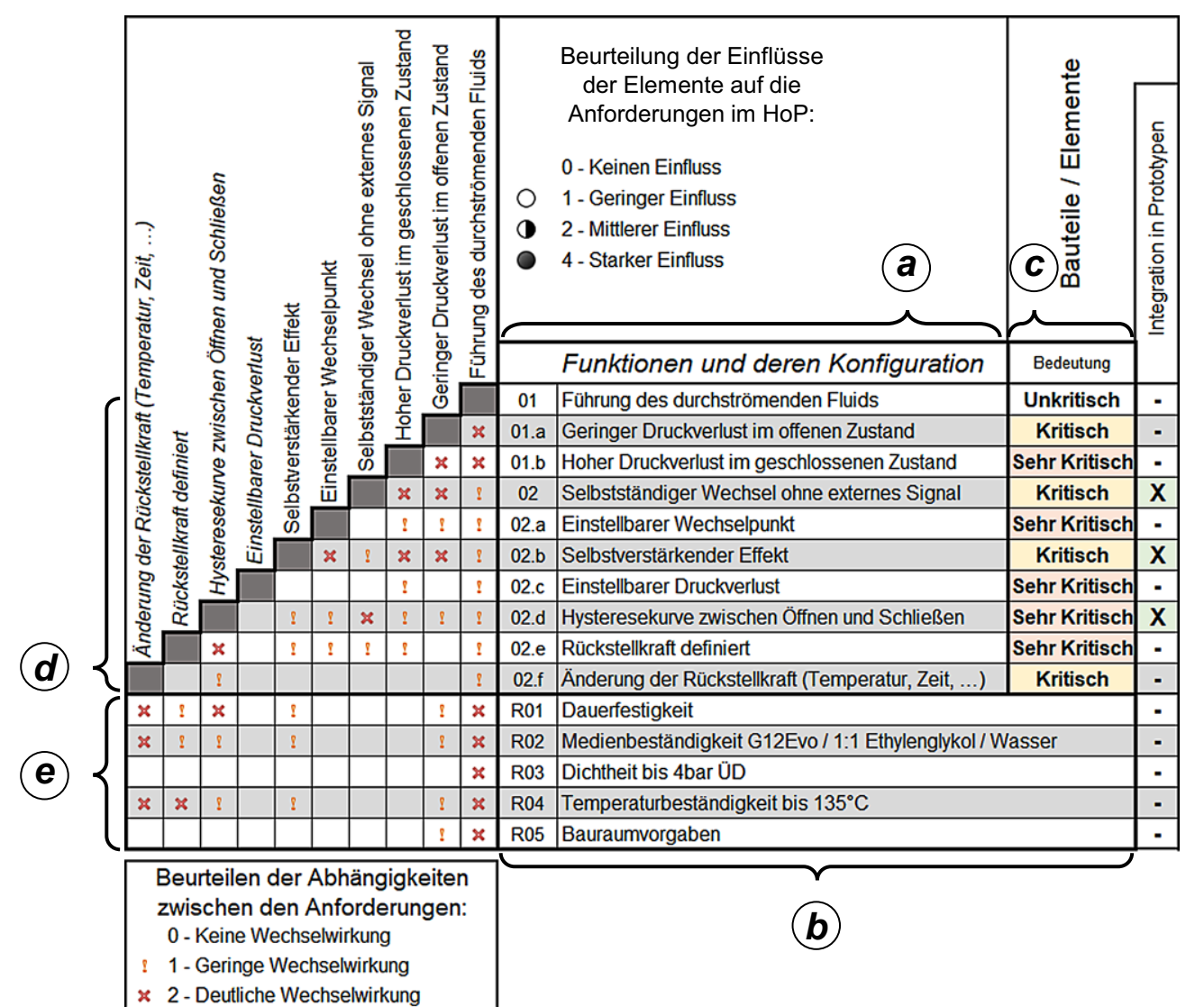

\begin{tabular}{|c|l|}
\hline$\Sigma$ & Summe der Einzelwertungen \\
\hline$\%$ & Einzelbedeutung der Funktionselemente für Gesamtprodukt \\
\hline $\mathrm{R}$ & Rang \\
\hline
\end{tabular}




\subsection{Einsatz virtueller oder physischer Prototypen}

Die Erkenntnisse aus dem House of Properties können in Verbindung mit verschiedenen, in der Literatur vorgestellten Vorgehen als Grundlage zur Entscheidung zwischen virtuellen und physischen Prototypen dienen [15, 16]. Das House of Properties gibt dann auch Aufschluss darüber, welche Anforderungen an ein Simulationsmodell gestellt werden müssen. Darüber hinaus kann abgeleitet werden, ob ein physischer Prototyp mit den verfügbaren Ressourcen und Herstellverfahren zielführend eingesetzt werden kann.

\subsection{Auswahl geeigneter additiver Herstellverfahren}

Die Bewertung der Bedeutung der Eigenschaften aus dem House of Properties kann auf die Auswahl geeigneter additiver Herstellverfahren für bestimmte Prototypen übernommen werden. Wie bereits erwähnt sollten Eigenschaften mit einer hohen funktionellen Bedeutung in einem physischen Prototyp entsprechend exakt abgebildet werden. Die Gruppe der additiven Fertigungsverfahren ist dabei sehr breit und umfasst verschiedene verwendbare Materialien und Prinzipien der Formgebung. Somit muss das additive Herstellverfahren derart gewählt werden, dass die entsprechend wichtigen Eigenschaftengeneriert werden können und mit den Eigenschaften des spritzgegossenen Bauteils übereinstimmen.

Für den gewählten Verwendungszweck kann im Beispiel die besondere Bedeutung einzelner Eigenschaften abgelesen werden. So ergibt sich eine unterschiedlich starke Gewichtung der Bauteileigenschaften. Eine hohe $\mathrm{HoP}$-Zahl deutet darauf hin, dass die entsprechende Eigenschaft mit hoher Präzision im Vergleich zu den realen Eigenschaften im Prototyp abgebildet werden muss.

Im aufgezeigten Beispiel sind für den gewünschten Verwendungszweck besonders die Werkstoffeigenschaften und die korrekte Abbildung der Geometrie des Bauteils von großer Bedeutung. Dies ist den Ergebnissen der Bewertung, die in Abb. 6 (unten) dargestellt sind, zu entnehmen. Es zeigt sich, dass besonders die Werkstoffeigenschaften und geometrischen Eigenschaften des Limiterelements hohen Einfluss auf die mit dem Öffnen und Schließen zusammenhängenden Funktionen (02, 02.b und 02.d) haben und daher im Prototyp möglichst nah am finalen Produkt abgebildet werden müssen. Entsprechend muss das Herstellverfahren für die Prototypen so ausgewählt werden, dass die verarbeitbaren Werkstoffe in den kritischen Eigenschaften denen des realen Bauteils ähneln und gleichzeitig die gesetzten Toleranzgrenzen eingehalten werden können. Die kritischen Werkstoffeigenschaften im Bauteil sind beispielsweise die maximal zulässige Dehnung, die Steifigkeit, die Zugfestigkeit und die Biegewechselfestigkeit. Die Bewertung des Einflusses der Eigenschaften auf die Funktio- nen des Bauteils findet auf der rechten Seite des House of Properties statt.

\subsection{Bewertung der Nutzbarkeit hergestellter Prototypen für weitere Untersuchungen}

Abschließend können die vorangegangenen Analysen auch dazu eingesetzt werden, die Nutzbarkeit bereits hergestellter Prototypen zur Untersuchung neuer Fragestellungen zu bewerten und auf diese Weise die Verlässlichkeit der Ergebnisse in der anschließenden Verifikation der Einhaltung der Anforderungen an Bauteilfunktionen zu bewerten. $\mathrm{Zu}$ dem kann eine Fehleranalyse darauf gestützt werden, in der zum einen Modellfehler und zum anderen Fehler im Bauteildesign identifiziert werden. Das House of Properties gibt außerdem Aufschluss darüber, ob frühere Verifikationsergebnisse weiterverwendet werden können, wenn beispielsweise der Werkstoff oder die Geometrie am Entwurf geändert werden. Wenn die Einhaltung der Anforderungen an bestimmte Funktionen bereits verifiziert wurde, kann rückwirkend ermittelt werden, ob durch die Änderung der Spezifikationen in der Konstruktion andere Ergebnisse zu erwarten sind.

Bauteile, deren Werkstoffeigenschaften beispielsweise keinen starken Einfluss auf die verifizierte Funktion haben, müssen gegebenenfalls nicht erneut überprüft werden, wenn sich eine Änderung der Werkstoffspezifikation ergibt. Somit trägt die Methode auch zu einer besseren Rückverfolgbarkeit und zur Vermeidung von unnötigen Wiederholungen bei.

\subsection{Beurteilung der Aussagegüte}

Die Beurteilung der Aussagegüte des Prototyps hilft bei der späteren Diskussion der Verifizierungsergebnisse. Ergeben sich bei der Verifizierung der Bauteileigenschaften Abweichungen des Prototyps von den in der Konstruktion festgelegten Anforderungen, so müssen diese nun hinsichtlich der Auswirkungen beurteilt werden. Auch hier kann man wiederum auf das House of Properties zugreifen. Wurden beispielsweise die Oberflächeneigenschaften eines Funktionselements durch Fertigungstoleranzen im Prototyp nicht korrekt abgebildet, kann man durch eine gegenläufige Betrachtung des House of Properties die Wechselwirkungen mit den Funktionen nachvollziehen. Dabei geht man von der entsprechenden Eigenschaft aus, welche die Soll-Spezifikation nicht erfüllen kann und liest ab, auf welche Funktionen sie welchen Einfluss hat. Durch diesen Rückschluss kann ein möglicher funktioneller Fehler bereits vor der eigentlichen Verifizierung erkannt werden. Je nach Grad der Abweichung vom Soll und der Einflussstärke kann somit das weitere Vorgehen diskutiert werden. Bei geringen Abweichungen sowie bei Bauteilen und Funktionselementen, 
welche im House of Properties mit einer niedrigen Bedeutung bewertet wurden, können die Prototypen dennoch verwendet werden. Allerdings sind die Ergebnisse entsprechend kritisch zu betrachten. Große oder besonders kritische Abweichungen können die Verwendbarkeit des Prototyps beziehungsweise der damit gewonnenen Ergebnisse stark einschränken oder ausschließen.

Durch den Abgleich der Eigenschaften sowie die objektive Bewertung dieser im House of Properties, können Verifikationsprozesse vermieden werden, deren Ergebnisse später nicht verlässlich sind. Hierdurch können finanzielle und zeitliche Ressourcen unmittelbar eingespart werden.

\section{Diskussion \& Fazit}

Zur Verifikation der Einhaltung von Anforderungen an Funktionen eines Produkts in Entwicklung mittels Prototypen muss sichergestellt sein, dass die Aussagefähigkeit des entwickelten Prototyps derart ausgeprägt ist, dass sich die Ergebnisse der Untersuchungen am Prototyp auch auf das Produkt in Entwicklung übertragen lassen. Das House of Properties bietet hierbei die Möglichkeit, den Zusammenhang zwischen den Funktionen des Produkts und dessen Eigenschaften herzustellen, sodass eine Entscheidungsgrundlage geschaffen wird, welche Eigenschaften des Produkts in Entwicklung im Prototyp abgebildet werden müssen und welche Eigenschaften von denen des späteren Produkts abweichen dürfen. Dadurch wird der Fokus auf die für die Verifikation ausschlaggebenden Eigenschaften gelegt und Herstellungsverfahren können entsprechend der geforderten Eigenschaften ausgewählt werden.

Gerade am beschriebenen Beispiel des Kunststoffspritzgussbauteils kann durch den Einsatz der Methode entschieden werden, welche additiven Verfahren zur Herstellung von Prototypen eingesetzt werden können. Dabei sind die Eigenheiten von Spritzgussbauteilen, wie Schwindung, Verzug oder anisotrope mechanische Eigenschaften zu beachten. Bei Produkten aus technischen Kunststoffen, die nahe der materialspezifischen Belastungsgrenzen belastet werden, ist die Modellierung und Analyse des Zusammenhangs zwischen Eigenschaften und Funktionen jedoch anfällig für Modellfehler und der Einfluss des Fertigungsverfahren hoch. Daher erscheint der Einsatz konventionell gefertigter Prototypen in diesem Bereich weiterhin als unumgänglich. Bei vereinfachten Verifikationen ist dieser sehr feine Detailgrad der Eigenschaftsanalyse jedoch meist nicht notwendig. Entsprechend konzentriert sich die Methode auf die Einflussstärke und somit auf die Vereinfachung von Prototypen unter Einhaltung lediglich der wichtigsten Eigenschaften.

Die entwickelte Methode und ihre Bausteine fügen sich vollständig in den allgemeinen Produktentwicklungszyklus nach VDI 2221 ein [5]. Dabei setzt sie kaum technische oder prozessuale Vorkenntnisse des Anwenders voraus, womit sie sehr einfach erlernbar und nachvollziehbar ist. Durch die aufgezeigten Analysen können Bedarfe für Prototypen unabhängig von der aktuellen Entwicklungsphase ermittelt und die Anforderungen an diese Prototypen definiert werden. Durch die offene Struktur der Methode lässt sie sich darüber hinaus auf weitere Branchen und Produkte anwenden.

Funding Open Access funding provided by Projekt DEAL.

Interessenkonflikt S. Schork, K. Güttinger und E. Kirchner geben an, dass kein Interessenkonflikt besteht.

Open Access Dieser Artikel wird unter der Creative Commons Namensnennung 4.0 International Lizenz veröffentlicht, welche die Nutzung, Vervielfältigung, Bearbeitung, Verbreitung und Wiedergabe in jeglichem Medium und Format erlaubt, sofern Sie den/die ursprünglichen Autor(en) und die Quelle ordnungsgemäß nennen, einen Link zur Creative Commons Lizenz beifügen und angeben, ob Änderungen vorgenommen wurden.

Die in diesem Artikel enthaltenen Bilder und sonstiges Drittmaterial unterliegen ebenfalls der genannten Creative Commons Lizenz, sofern sich aus der Abbildungslegende nichts anderes ergibt. Sofern das betreffende Material nicht unter der genannten Creative Commons Lizenz steht und die betreffende Handlung nicht nach gesetzlichen Vorschriften erlaubt ist, ist für die oben aufgeführten Weiterverwendungen des Materials die Einwilligung des jeweiligen Rechteinhabers einzuholen.

Weitere Details zur Lizenz entnehmen Sie bitte der Lizenzinformation auf http://creativecommons.org/licenses/by/4.0/deed.de.

\section{Literatur}

1. Hopmann C, Menges G, Michaeli W, Mohren P (2018) Spritzgießwerkzeuge. Auslegung, Bau, Anwendung, 7. Aufl. Hanser, München

2. Ehrlenspiel K, Kiewert A, Lindemann U, Mörtl M (2014) Kostengünstig Entwickeln und Konstruieren. Kostenmanagement bei der integrierten Produktentwicklung, 7. Aufl. VDI-Buch. Springer Vieweg, Berlin

3. Johannaber F, Michaeli W (2014) Handbuch Spritzgießen, 2. Aufl. Hanser, München

4. Graner M (2013) Der Einsatz von Methoden in Produktentwicklungsprojekten. Eine empirische Untersuchung der Rahmenbedingungen und Auswirkungen. Springer Gabler, Wiesbaden

5. VDI Richtlinie 2221 (2019-11) Entwicklung technischer Produkte und Systeme Modell der Produktentwicklung. Beuth, Düsseldorf

6. Schork S, Kirchner E (2018) Defining requirements in prototyping: the holistic prototype and process development. In: Ekströmer P, Schütte S, Ölvander J (Hrsg) Proceedings of NordDesign 2018 NordDESIGN.

7. Albers A, Klingler S, Wagner D (2014) Prioritization of validation activities in product development processes. In: Marjanović D, Štorga M, Pavković N, Bojčetić N (Hrsg) Proceedings of the DESIGN 2014 13th International Design Conference. The Design Society, Zagreb, S 81-90

8. DIN EN ISO 10209:2012-11 (2012) Technische Produktdokumentation; Vokabular; Begriffe für technische Zeichnungen, Produktdefinition und verwandte Dokumentation. Beuth, Berlin 
9. VDI Richtlinie 2223 (2004) Methodisches Entwerfen technischer Produkte, ICS 03.100.40. Beuth, Düsseldorf

10. Weber C, Werner H (2001) Schlußfolgerungen für „Design for X“ (DfX) aus der Perspektive eines neuen Ansatzes zur Modellierung von Produkten und Produktentwicklungsprozessen. In: Meerkamm H (Hrsg) Design for X. Beiträge zum 12. Symposium Design for X. Lehrstuhl für Konstruktionstechnik Univ. ErlangenNürnberg, Erlangen

11. Albers A, Matthiesen S (2002) Konstruktionsmethodisches Grundmodell zum Zusammenhang von Gestalt und Funktion technischer Systeme. Konstruktion 54(7/8):55-60

12. Niemann G, Winter H, Höhn B-R, Stahl K (2019) Maschinenelemente 1. Konstruktion und Berechnung von Verbindungen, Lagern, Wellen, 5. Aufl. Springer, Berlin, Heidelberg
13. DIN Deutsches Institut für Normung e.V. (2015) „Qualitätsmanagementsysteme - Grundlagen und Begriffe, “ 01.040.01;03.120.10, DIN EN ISO 9000. Beuth, Berlin

14. Feldhusen J, Grote K-H (Hrsg) (2013) Pahl/Beitz Konstruktionslehre. Methoden und Anwendung erfolgreicher Produktentwicklung, 8. Aufl. Springer Vieweg, Berlin, Heidelberg

15. Hamon C, Green M, Dunlap B, Camburn B, Crawford R, Jensen D (2014) Virtual or physical prototypes? Development and testing of a prototyping planning tool, ASEE Annual Conference and Exposition, Conference Proceedings, Indianapolis

16. Filippi S, Barattin D (2014) A selection algorithm for prototyping activities. Int J Interact Des Manuf 8(1):1-11. https://doi.org/10. 1007/s12008-013-0189-9 